\title{
Differences in metabolic profiles between bicuspid and tricuspid aortic stenosis in the setting of transcatheter aortic valve replacement
}

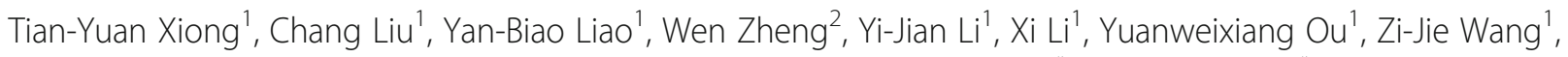
Xi Wang ${ }^{1}$, Chang-Ming Li ${ }^{1}$, Zhen-Gang Zhao ${ }^{1}$, Yuan Feng ${ }^{1}$, Xiao-Jing Liu ${ }^{2,3^{*}}$ and Mao Chen ${ }^{1 *}$

\begin{abstract}
Background: To explore why bicuspid aortic stenosis has certain clinical differences from the tricuspid morphology, we evaluated the metabolomics profile involved in bicuspid aortic valve (BAV) aortic stenosis prior to and after transcatheter aortic valve replacement (TAVR) in comparison with tricuspid aortic valve (TAV).

Methods: In this TAVR cohort with prospectively collected data, blood samples were obtained before TAVR valve deployment and at the 7th day after TAVR, which were then sent for liquid and gas chromatography-mass spectrometry detection. Besides comparisons between BAV and TAV, BAV patients were also divided in subgroups according to baseline hemodynamics (i.e. maximal transaortic velocity, $V_{\max }$ ) and post-procedural reverse left ventricular (LV) remodeling (i.e. the change in LV mass index from baseline, $\Delta$ LVMI) for further analysis. Metabolic differences between groups were identified by integrating univariate test, multivariate analysis and weighted correlation network analysis algorithm.
\end{abstract}

Results: A total of 57 patients were enrolled including 33 BAV patients. The BAV group showed lower arginine and proline metabolism both before and post TAVR than TAV represented by decreased expression of L-Glutamine. In BAV subgroup analysis, patients with baseline $V_{\max }>5 \mathrm{~m} / \mathrm{s}(n=11)$ or the $4^{\text {th }}$ quartile of change in $\triangle \mathrm{LVMI}$ at oneyear follow-up (i.e. poorly-recovered $L V, n=8$ ) showed elevated arachidonic acid metabolism compared with $V_{\max }<4.5 \mathrm{~m} / \mathrm{s}(n=12)$ or the $1^{\text {st }}$ quartile of $\Delta L V M I$ (i.e. well-recovered $\left.L V, n=8\right)$ respectively.

Conclusions: Difference in arginine and proline metabolism was identified between BAV and TAV in TAVR recipients. Elevated arachidonic acid metabolism may reflect more severe baseline hemodynamics and worse LV reserve remodeling after TAVR in BAV.

Keywords: Bicuspid aortic valve, Aortic stenosis, Metabolomics, Transcatheter aortic valve replacement, Inflammation

\footnotetext{
* Correspondence: liuxq@scu.edu.cn; maochen@pcrccv.com

${ }^{2}$ Laboratory of Mitochondrial Biology, West China-Washington Mitochondria and Metabolism Center, West China Hospital, Sichuan University, Chengdu, People's Republic of China

'Department of Cardiology, West China Hospital, Sichuan University, \#37 Guo Xue Alley, Chengdu 610041, People's Republic of China

Full list of author information is available at the end of the article
}

(c) The Author(s). 2020 Open Access This article is licensed under a Creative Commons Attribution 4.0 International License, which permits use, sharing, adaptation, distribution and reproduction in any medium or format, as long as you give appropriate credit to the original author(s) and the source, provide a link to the Creative Commons licence, and indicate if changes were made. The images or other third party material in this article are included in the article's Creative Commons licence, unless indicated otherwise in a credit line to the material. If material is not included in the article's Creative Commons licence and your intended use is not permitted by statutory regulation or exceeds the permitted use, you will need to obtain permission directly from the copyright holder. To view a copy of this licence, visit http://creativecommons.org/licenses/by/4.0/ The Creative Commons Public Domain Dedication waiver (http://creativecommons.org/publicdomain/zero/1.0/) applies to the data made available in this article, unless otherwise stated in a credit line to the data. 


\section{Background}

Bicuspid aortic valve (BAV) is the most common congenital defect characterized by two leaflets in the aortic position, which may have a spectrum of clinical presentations $[1,2]$. In terms of aortic stenosis, some interesting distinctions have been observed in BAV patients. Compared with normally formed tricuspid aortic valve (TAV), BAV patients often develop more severe stenosis with heavier calcification and/or fibrosis in aortic leaflets at a younger age [3]. The difference is not limited to the valve itself, but also seems to involve the left ventricle (LV). Patients with BAV were reported to undergo less pronounced reverse LV remodeling than TAV [4] after definitive treatment by means of surgical or transcatheter aortic valve replacement (TAVR). Despite these observations indicating bicuspid aortic stenosis to have its own characteristics, current follow-up and treatment of bicuspid aortic stenosis do not differ from TAV patients.

A handful of studies have indeed identified some intrinsic genetic bases engaged in the etiology of BAV, such as the gene of ACTA2 [5] and NOTCH1 [6]. However, as the final stage in processing genomic information, metabolites were considered to significantly influence cellular activity and directly reflect extracellular microenvironment $[7,8]$. Thus, metabolomics profiling technologies [9] are more likely to determine novel disease biomarkers and provide insights in biological mechanisms, which would be essential to improve treatment strategies. To date, few studies have focused on the specific metabolic mechanisms involved in bicuspid aortic stenosis, especially after aortic valve replacement.

In this prospective study, we sought to incorporate metabolomics profiling technologies in the setting of TAVR, with patients of both bicuspid and tricuspid morphology. In particular, detailed analyses are planned for BAV patients to explore potential metabolomic differences in subgroups with worse baseline hemodynamics and poorer LV recovery post-TAVR.

\section{Methods}

\section{Study population}

From November 2015 to August 2016, patients with severe symptomatic aortic stenosis, who successfully underwent TAVR in our center with both pre- and postprocedural blood samples collected, were enrolled in the study, while patients who received TAVR due to failed bioprostheses were excluded ( $N=57,32$ females). Patients were divided into BAV and TAV groups according to their valve morphology confirmed and classified on multi-slice computed tomography (MSCT). TAVR was performed by the same heart team from the transfemoral access under general anesthesia, as described previously [10]. All enrolled patients received the same management process and were treated with the same intervening protocol. Participants enrolled in this study signed the informed consent in accordance with the Declaration of Helsinki. This study was approved by the Institutional Review Board of West China Hospital, Sichuan University.

\section{Echocardiography follow-up}

Transthoracic echocardiography (TTE) were arranged before discharge and at routine follow-ups at the 1st month, 3rd month, $6^{\text {th }}$ month and 1st year after TAVR. To assess reverse LV remodeling after TAVR, LV mass (LVM) and LVM index (LVMI) were calculated [11]. The change in LVM and LVMI was calculated as the value at follow-ups subtracted the value at baseline (shown as $\triangle \mathrm{LVM}$ and $\triangle \mathrm{LVMI}$ ) and served as a surrogate endpoint to LV recovery post-TAVR.

\section{Blood sample collection and processing}

Blood sample was obtained from peripheral vein before valve deployment during the index TAVR procedure and at the 7th day after TAVR (normally the day of discharge) in K2EDTA-treated tubes (BD). Subsequently, these blood samples were centrifuged at $2000 \mathrm{~g}$ for 10 min to pellet the cellular elements. The supernatant plasma was stored at $-80^{\circ} \mathrm{C}$ until sample preparation for liquid chromatography-mass spectrometry (LC-MS) and gas chromatography-mass spectrometry (GC-MS) analysis.

\section{Metabolomics detection}

Metabolomics profiling was detected and processed by Chinese Academy of Sciences in Dalian based on methods described in previous studies [12, 13]. Briefly, metabolomic analysis was conducted on ACQUITY Ultra Performance Liquid Chromatography (UPLC, Waters Corporation, Manchester, UK) system with ACQUITY UPLC BEH C8 $1.7 \mu \mathrm{m}(2.1 \times 100 \mathrm{~mm})$ column (Waters, Milford, MA) for positive ion metabolites separation setting at $50^{\circ} \mathrm{C}$ and ACQUITY UPLC HSS T3 $1.8 \mu \mathrm{m}(2.1 \times 100 \mathrm{~mm})$ column (Waters, Milford, MA) for negative ion metabolites separation setting at $50^{\circ} \mathrm{C}$. A QP 2010 Plus GC-MS system (Shimadzu, Japan) with a DB-5MS $(30 \mathrm{~m} \times 250 \mu \mathrm{m} \times 0.25 \mu \mathrm{m}$, Agilent Technologies, USA) was used in GC-MS analysis. The procedures were performed in accordance with the manufacturers' protocols of all devices. Then, raw data were converted to mzData formats via Agilent MassHunter Qualitative software (Agilent, Santa Clara, CA, USA). The program XCMS (version 1.40.0) (https:// xcmsonline.scripps.edu/) was used to preprocess the raw data, with the default parameters. In the quality control (QC) process, nearly $100 \%$ features in QC samples had relative standard deviation (RSD) distribution less than $30 \%$, and were within 3SD, which demonstrated a 
satisfactory data quality. The resulting matrix was constructed by retention time, mass-to-charge ratio $(\mathrm{m} / \mathrm{z})$, and normalized ion intensities.

\section{Metabolomics interpretation}

The model of partial least squares discriminant analysis (PLS-DA) was used to classify metabolic characteristics of bicuspid aortic stenosis from tricuspid aortic stenosis. The variable importance project (VIP) from PLS-DA was also calculated. Furthermore, $p$-value from t-test analysis and fold-change value (FC) were executed to discover differentially expressed metabolites, which were exhibited by the volcano plot. With a pre-specified cutoff of $p$-value $<0.05, \mathrm{FC}>1.2$ and VIP $>1.2$, the dominant molecules were to be identified. These analyses were completed with an in-house script by $\mathrm{R}$ platform (https://www.r-project.org).

To futher identify the essential metabolic pathways and hub metabolites engaged in phenotypic alterations, pathway enrichment analysis was employed to summarize more interpretable results [14]. However, if the number of metabolites identified above was over 100, weighted gene coexpression network analysis (WGCNA) algorithm was to be first utilized to narrow down the number of metabolites sent to pathway enrichment analysis [15]. This protocol enabled the detection of hub molecules which were also correlated with clinical traits. Metabolites to be sent to patheway enrichment analysis were then selected by Venn diagram of both hub molecules defined by the WGCNA protocol and differentially expressed metabolites defined by the volcano plot. Pathway enrichment analysis was conducted with MetaboAnalyst platform (http://www.metaboanalyst.ca) [16].

\section{Subgroup analysis}

For the subgroup analysis to explore the specific metabolites related to worse hemodynamics in BAV patients before TAVR, $4 \mathrm{~m} / \mathrm{s}<$ maximal transaortic velocity $\left(\mathrm{V}_{\max }\right)<$ $4.5 \mathrm{~m} / \mathrm{s}$ was defined as the lower $V_{\max }$ group, whereas $\mathrm{V}_{\max }>5 \mathrm{~m} / \mathrm{s}$ was defined as the higher $\mathrm{V}_{\max }$ group. Postprocedurally, the value of $\triangle \mathrm{LVMI}$ at 1 year was divided into quartiles. BAV patients in the $4^{\text {th }}$ quartile were regarded to have the worst LV recovery, while those in the $1^{\text {st }}$ quartile were regarded to have the best LV recovery.

\section{Statistical analysis}

Normality was assessed for all clinical datasets by the Shapiro-Wilk's test. Continuous results were presented as the mean $\pm \mathrm{SD}$ or median (IQR) according to their distribution. Independent $\mathrm{t}$-test (for variables that were normally distributed) or Mann-Whitney U-test (for variables that

Table 1 Clinical features of enrolled patients

\begin{tabular}{|c|c|c|c|}
\hline & Bicuspid aortic stenosis $(n=33)$ & Tricuspid aortic stenosis $(n=24)$ & $P$-value \\
\hline Female, n (\%) & $24(72.73 \%)$ & $8(33.33 \%)$ & 0.006 \\
\hline Age (years) & $72.48 \pm 5.62$ & $74.38 \pm 5.53$ & 0.21 \\
\hline Height (m) & $1.57 \pm 0.08$ & $1.61 \pm 0.09$ & 0.12 \\
\hline Weight (kg) & $55.15 \pm 9.88$ & $59.69 \pm 9.87$ & 0.92 \\
\hline BMI $\left(\mathrm{kg} / \mathrm{m}^{2}\right)$ & $22.13 \pm 3.45$ & $22.83 \pm 3.23$ & 0.44 \\
\hline STS-PROM (\%) & $6.50(4.42-8.59)$ & $8.26(4.93-13.38)$ & 0.09 \\
\hline \multicolumn{4}{|l|}{ Comorbidities } \\
\hline Hypertension, n(\%) & $15(45.45 \%)$ & $7(29.17 \%)$ & 0.27 \\
\hline Diabetes, n (\%) & $1(3.03 \%)$ & $3(12.5 \%)$ & 0.15 \\
\hline Chronic lung disease, n (\%) & $18(54.55 \%)$ & $19(79.17 \%)$ & 0.09 \\
\hline Coronary artery disease, n (\%) & $16(48.48 \%)$ & $10(41.67 \%)$ & 0.78 \\
\hline Prior myocardial infarction, n (\%) & 0 & 0 & - \\
\hline Prior percutaneous coronary intervention, n (\%) & $2(6.06 \%)$ & $3(12.5 \%)$ & 0.64 \\
\hline Peripheral vascular disease, n (\%) & $20(60.60 \%)$ & $20(83.3 \%)$ & 0.08 \\
\hline Chronic kidney disease, n (\%) & $6(18.18 \%)$ & $5(20.83 \%)$ & 0.99 \\
\hline \multicolumn{4}{|l|}{ Baseline echocardiography } \\
\hline Left ventricular ejection fraction, $\%$ & $53.73 \pm 15.05$ & $44.67 \pm 14.19$ & 0.025 \\
\hline Transaortic $V_{\max }, \mathrm{m} / \mathrm{s}$ & $5.14 \pm 0.78$ & $4.57 \pm 0.85$ & 0.011 \\
\hline Transaortic $\mathrm{PG}_{\text {mean }}, \mathrm{mmHg}$ & $67.52 \pm 21.99$ & $55.79 \pm 16.01$ & 0.031 \\
\hline LVM, g & $265.35 \pm 87.38$ & $338.84 \pm 98.46$ & 0.004 \\
\hline LVMI, $\mathrm{g} / \mathrm{m}^{2}$ & $161.66 \pm 48.78$ & $197.66 \pm 62.32$ & 0.018 \\
\hline Ascending aorta diameter, $\mathrm{mm}$ & $40.50 \pm 5.59$ & $38.75 \pm 4.32$ & 0.21 \\
\hline
\end{tabular}




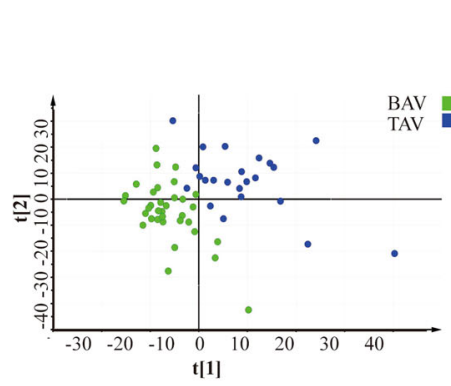

(A)

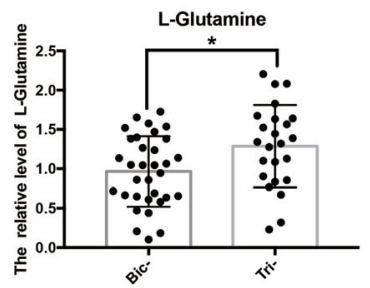

Pyrrole-2-carboxylic acid

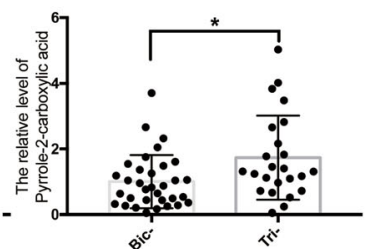

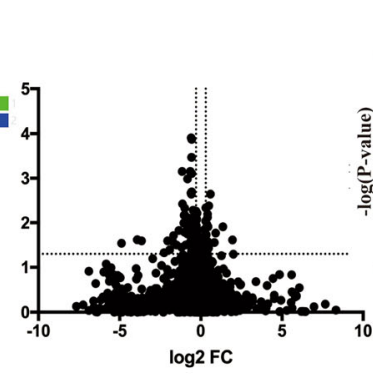

(B)
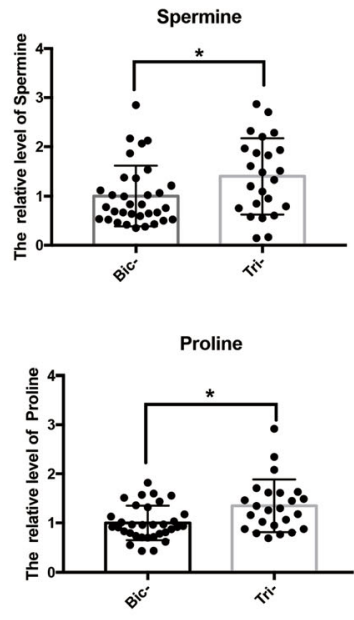

(D)

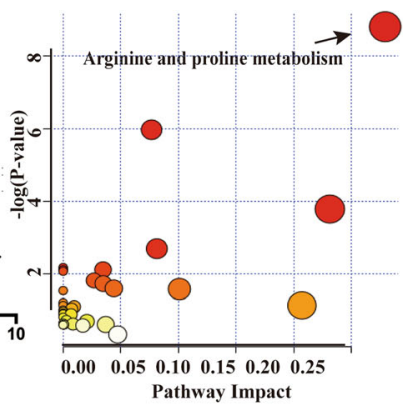

(C)
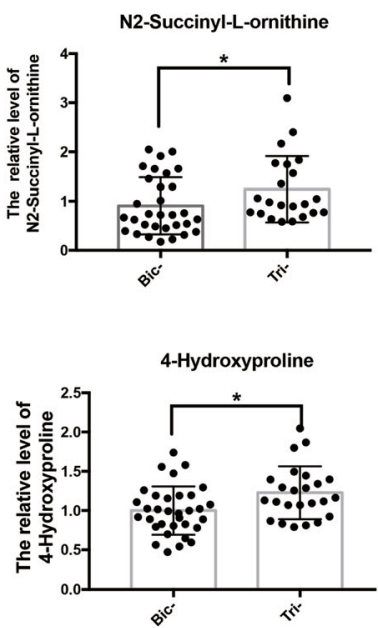

Fig. 1 The specific metabolites differentially expressed between BAV and TAV patients prior to TAVR. a PLS-DA analysis implied the diverse metabolic patterns between bicuspid aortic stenosis and tricuspid aortic stenosis. b Comparison of all metabolites between BAV and TAV by volcano plot. c Enrichment analysis for these dominant metabolites which separated BAV stenosis participants from TAV stenosis ones ( $p$-value $<$ 0.05). $\mathbf{d}$ The expression of metabolites in arginine and proline metabolism pathway between BAV and TAV patients before TAVR (* indicated $p$-value $<0.05$ )

were not normally distributed) was carried out to assess differences in clinical features between individuals in two groups. A two-tailed $p$ value $<0.05$ was considered as significant.

\section{Results}

\section{Clinical features of study cohort}

A total of 57 patients were enrolled with blood sample collected at the two time points. Baseline was summarized in Table 1. Confirmed with MSCT, there were 33 patients with BAV in this cohort. The average age was similar between BAV and TAV groups (72.48 \pm 5.62 vs. $74.38 \pm 5.53$ years, $P=0.21$ ). Two patients were low-flow, low-gradient aortic stenosis with reduced ejection fraction, while others were high-gradient aortic stenosis. BAV patients had higher $\mathrm{V}_{\max }(5.14 \pm 0.78$ vs. $4.57 \pm 0.85 \mathrm{~m} / \mathrm{s}, P=0.01)$ but lower LVM (265.35 [87.38] vs. $338.84[98.46] \mathrm{g}, P<0.01)$ than TAV patients on pre-

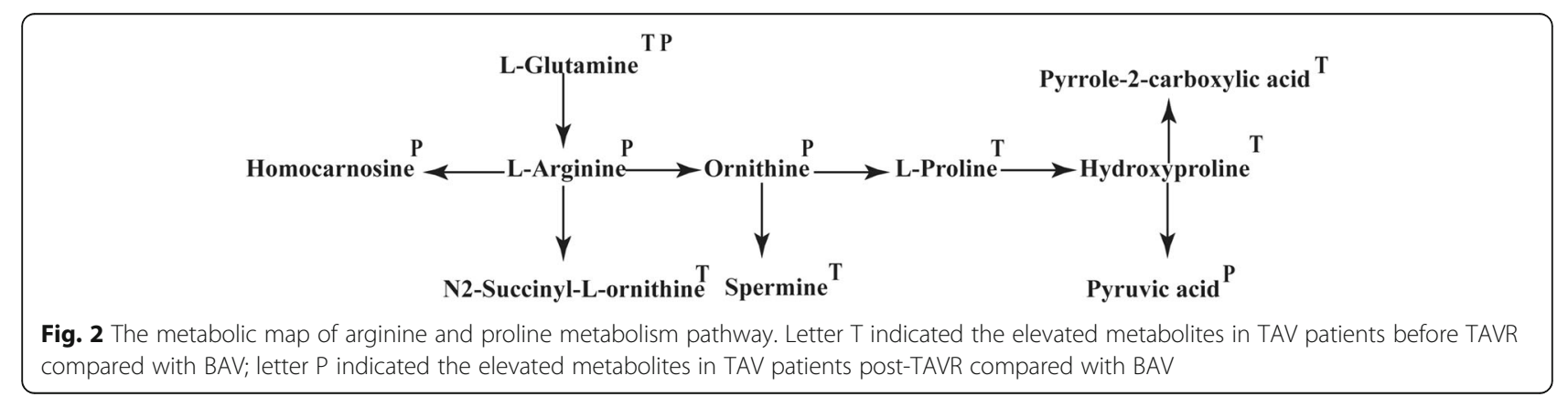


procedural TTE. Calcium volume of the aortic valve measured on MSCT was similar between BAV and TAV patients (705.70 [485.15] vs. $535.25[598.93] \mathrm{m}^{3}, P=$ 0.27).

Hemodynamics and reverse LV remodeling postTAVR were illustrated in Supplementary Figure 1. The value of post-procedural $\mathrm{V}_{\max }$, LVM and LVMI were comparable between the two groups through one-year follow-up, but BAV patients experienced less pronounced reverse LV remodeling than TAV patients ( $\triangle$ LVMI, $-35.35 \pm 42.07$ vs. $\left.-68.4 \pm 56.89 \mathrm{~g} / \mathrm{m}^{2}, P=0.03\right)$.

\section{Distinctions of arginine and proline metabolism pathway} between patients with BAV and TAV

Prior to TAVR, a diverse metabolic pattern of BAV from TAV was observed through the separation on PLS-DA score plot (Fig. 1a). By global metabolomics profiling, the differentially expressed metabolites of BAV from
TAV were further identified with the cutoff of $P$-value $<$ 0.05, FC-value $>1.2$ and VIP value $>1.2$ (Fig. 1b). According to the enrichment analysis, obviously altered metabolites were mapped to the arginine and proline metabolism pathway (Fig. 1c). In details, the expression of 6 metabolites in this pathway, namely L-Glutamine, L-Proline, Hydroxyproline, Pyrrole-2-carboxylic acid, N2-Succinyl-L-ornithine and spermine, was significantly lower in BAV than TAV (Fig. 1d). The locations of these remarkably altered molecules were further elucidated in the metabolic map of the arginine and proline metabolism pathway and marked with the letter T in Fig. 2.

Seven days post-TAVR, the metabolic pattern still separated between BAV and TAV groups on PLS-DA model (Fig. 3a). With the same cutoff of $P$-value, FC and VIP, differentially expressed molecules between the two groups were identified and shown by the volcano plot (Fig. 3b). On the basis of enrichment analysis, these

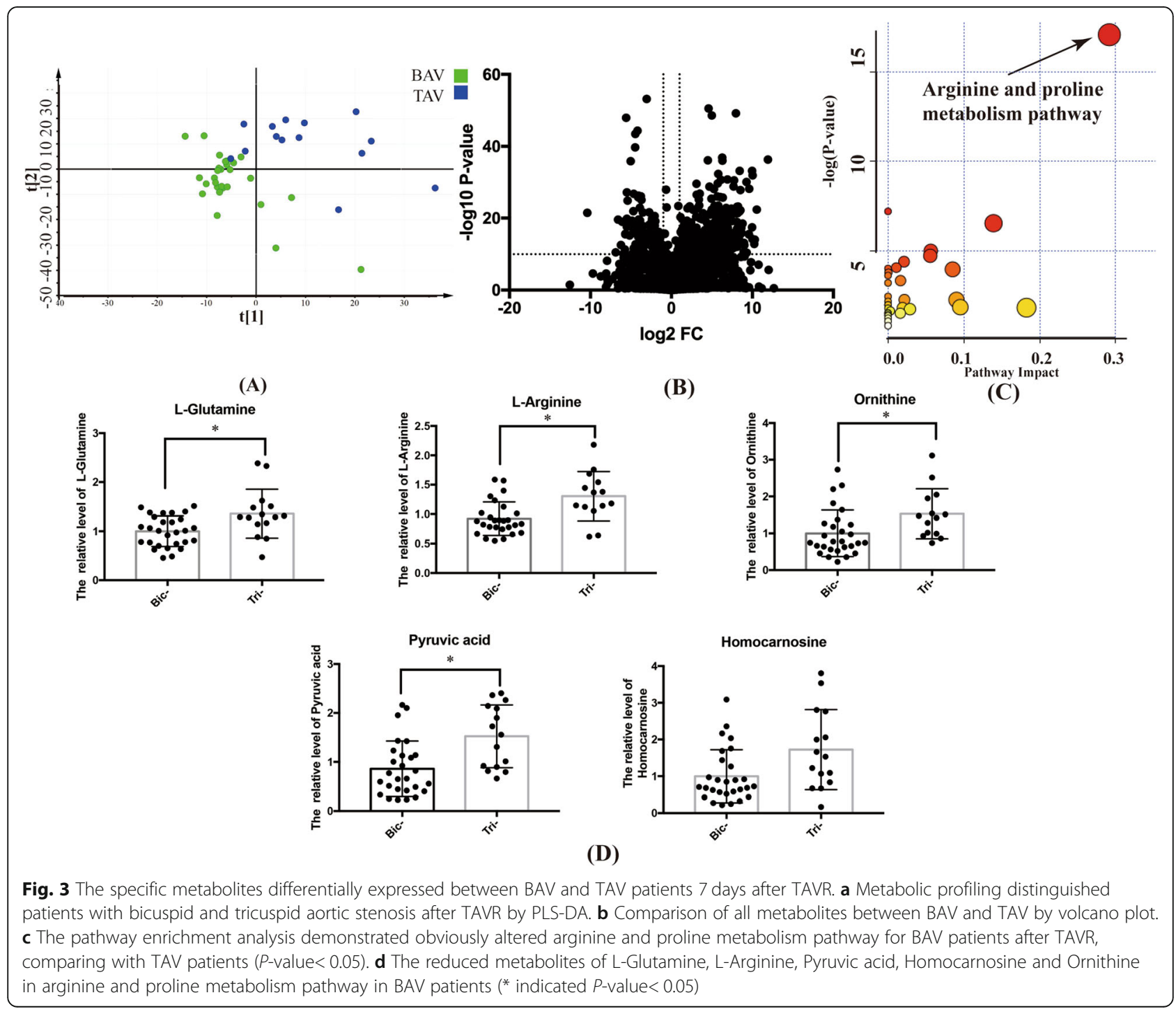



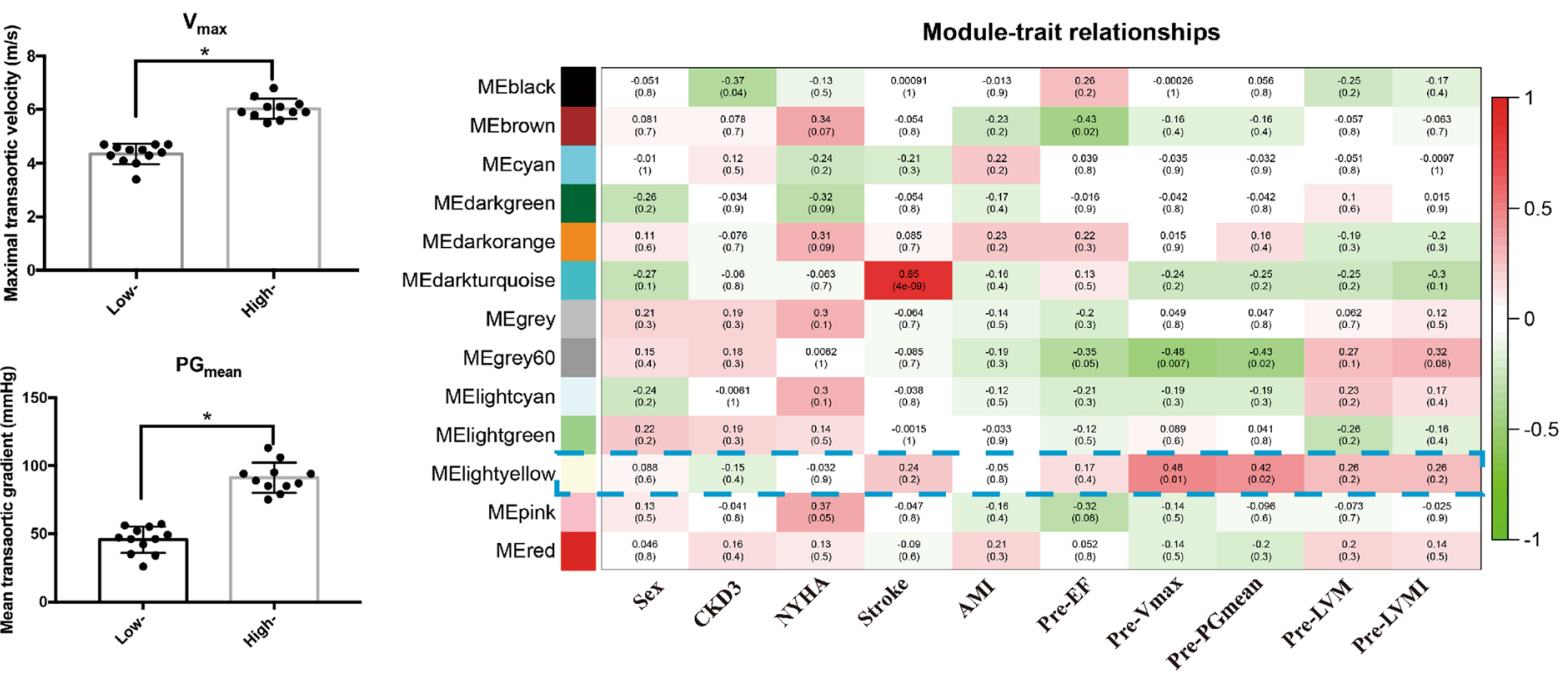

(A)

(C)

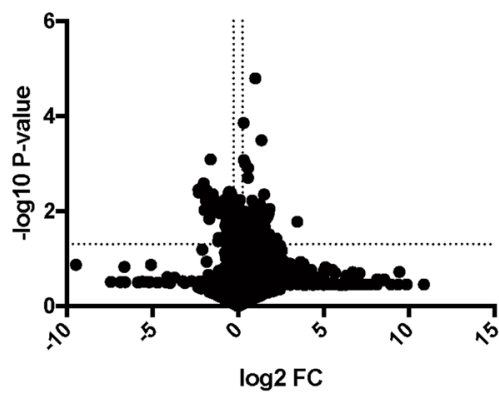

(B)

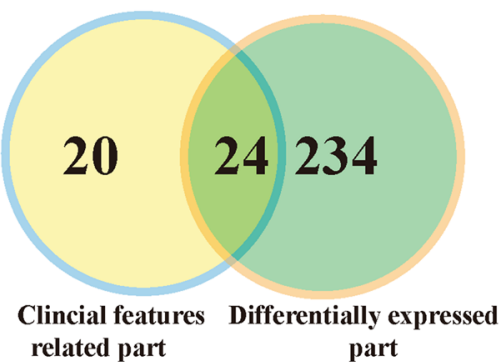

(D)

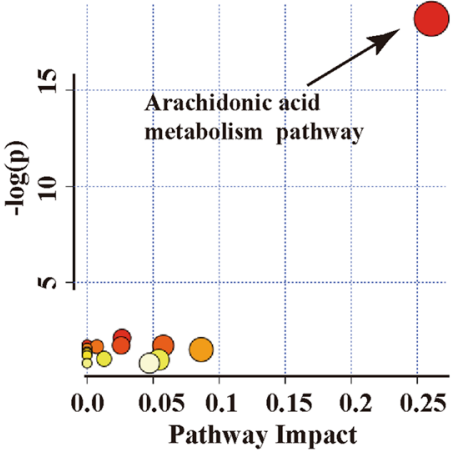

(E)
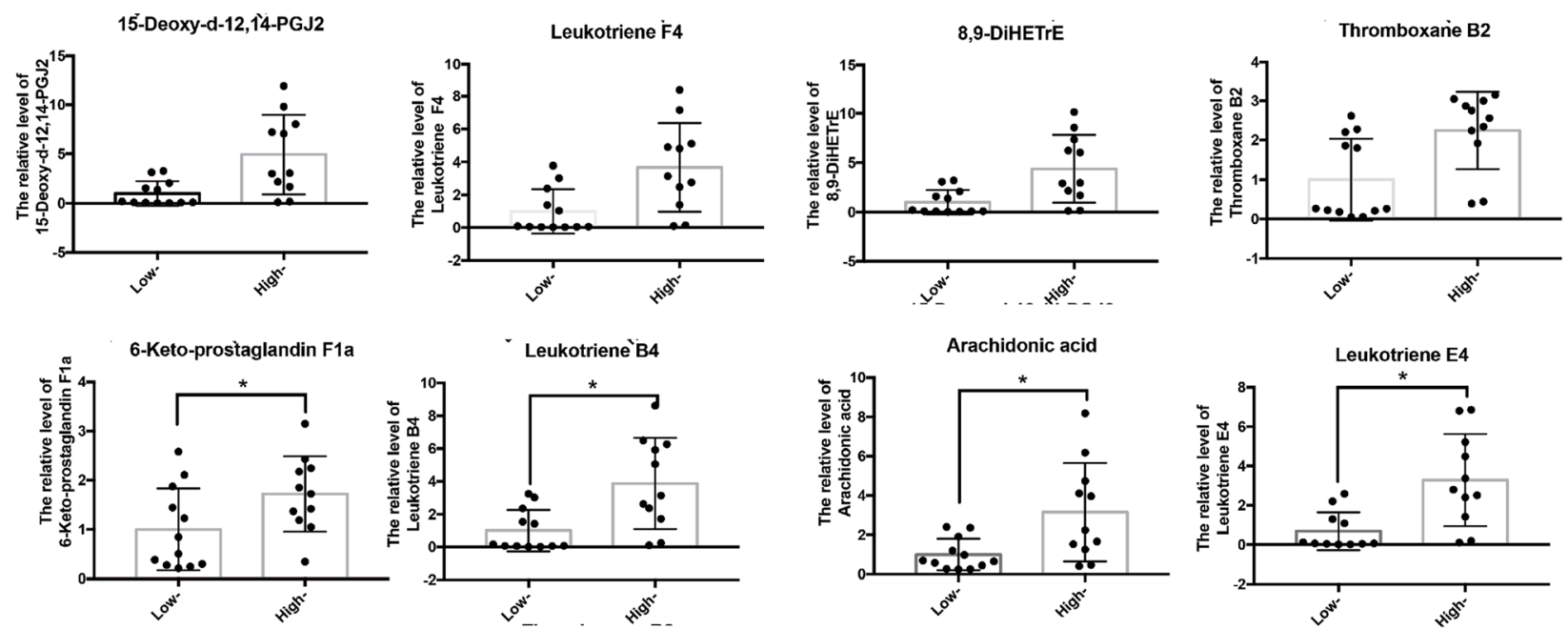

(F)

Fig. 4 Baseline subgroup analysis for BAV patients. a Baseline hemodynamics in lower and higher baseline $V_{\max } g r o u p s .{ }^{*}$ indicated $p$-value $\left.<0.05\right)$. b Comparison of all metabolites between the lower and higher $V_{\max }$ groups by volcano plot. $\mathbf{c}$ WGCNA algorithm identified the metabolic module closely related to baseline $V_{\max }$. $\mathbf{d}$ Venn plot indicated the impact metabolites which both were positively related to baseline $V_{\max }$ and differentially expressed between BAV and TAV. e Pathway enrichment analysis detected the significantly altered arachidonic acid metabolism pathway $(p$-value $<0.05)$. f The different expression of metabolites in arachidonic acid metabolism pathway between patients with lower and higher baseline $V_{\max }{ }^{*}$ indicated $p$-value $<0.05$ ) 
identified metabolites were mapped, again, to the arginine and proline metabolism pathway (Fig. 3c). The expression of 5 metabolites in this pathway, namely LGlutamine, L-Arginine, Pyruvic acid, Homocarnosine and Ornithine, was significantly lower in BAV than TAV (Fig. 3d). In the metabolic map of the arginine and proline metabolism pathway, they were marked with the letter $\mathrm{P}$ in Fig. 2.

\section{Subgroup analysis of BAV}

At baseline, $12 \mathrm{BAV}$ patients were identified as the lower $\left(4 \mathrm{~m} / \mathrm{s}<\mathrm{V}_{\max }<4.5 \mathrm{~m} / \mathrm{s}\right)$ and 11 as the higher $\left(\mathrm{V}_{\max }>5\right.$ $\mathrm{m} / \mathrm{s}) \mathrm{V}_{\max }$ group respectively. Details were shown in Fig. 4a. By univariate and multivariate analysis, we identified 258 differentially expressed metabolites between these two groups (Fig. 4b). WGCNA algorithm revealed hub molecules correlated with pre-procedural $\mathrm{V}_{\max }$ and $\mathrm{PG}_{\text {mean }}$, i.e. the MElightyellow module (labeled in blue frame) (Fig. 4c). A total of 24 hub metabolites were determined (Fig. 4d) and then mapped to the arachidonic acid metabolism pathway by enrichment analysis (Fig. 4e). In details, 8 metabolites including 6-Ketoprostaglandin F1a, Leukotriene B4, Arachidonic acid, Leukotriene E4, and etc., expressed significantly less in the lower than higher $V_{\max }$ group (Fig. 4f). The location of these molecules was listed in the metabolic map of the arachidonic acid metabolism pathway, which were labeled by letter V in Fig. 5 .

At one-year follow-up, 8 patients within the $4^{\text {th }}$ quartile of $\triangle$ LVMI value were identified as the poorlyrecovered group and 8 patients within the $1^{\text {st }}$ quartile of $\triangle$ LVMI value as the well-recovered group (Fig. 6a and b). By vocalno plot, 37 differentially expressed metabolites in these two groups negatively related to $\triangle \mathrm{LVMI}$ were detected (Fig. 6c) and mapped, again, to the arachidonic acid metabolism pathway (Fig. 6d). The metabolites of 15-KETE, 15(S)-HETE, arachidonic acid, prostaglandin G2, Thromboxane B2, Leukotriene A4 and Leukotriene B4 were significantly increased in patients in the poorly-recovered group (Fig. 6e). Their locations were listed in in Fig. 5.

\section{Discussion}

The incorporation of metabolomics profiling technologies to TAVR patients offered us a unique way to explore bicuspid aortic stenosis in comparison with the normal tricuspid morphology, both before and after the stenotic status been corrected. The two major findings of this study are (1) BAV patients had an altered arginine and proline metabolism pathway compared with TAV, which sustained post-TAVR; (2) the arachidonic acid metabolism pathway seems to be associated with more severely affected hemodynamics, as well as poorlyrecovered LV post-TAVR in BAV. These preliminary results provide novel insights to further elucidate the different disease spectrum of bicuspid aortic stenosis and determine possible biomarkers for monitoring progression and prognosis of bicuspid aortic stenosis.

Patients with bicuspid aortic stenosis are known to require aortic valve replacement at a much younger age than their tricuspid counterparts, usually within productive years of life [17]. As we currently do not have means to effectively postpone the disease progress of aortic stenosis, determining the difference between bicuspid and tricuspid aortic stenosis may be an alternative way for BAV patients to at least face this inevitable intervention later in their life. Elevated arginine and proline metabolism pathway was found in TAV compared with BAV, regardless whether severe aortic stenosis was corrected or not. This finding suggested that metabolites in the arginine and proline pathway may be potential targets to delay the progression of bicuspid aortic stenosis. The arginine and proline metabolism pathway generates nitric oxide with Nitric Oxide Synthase (NOS) [18, 19]. Elevated endothelial nitric oxide synthase (eNOS) has been found in TAV [20], but eNOS was low expressed and irregularly distributed in BAV [21]. ENOS is essential in regulating biological homeostasis. Limited eNOS reduces cellular NO level which could lead to

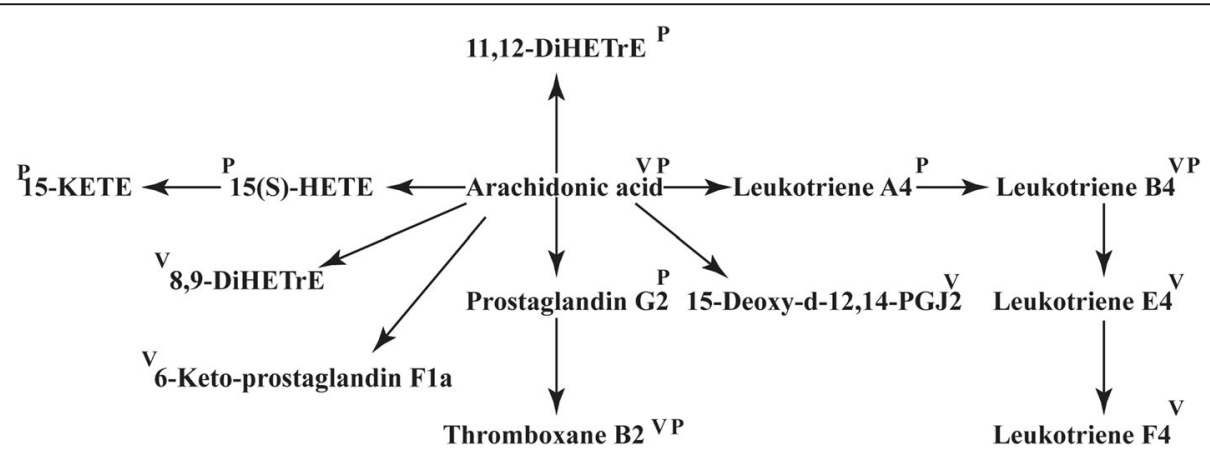

Fig. 5 The metabolic map of arachidonic acid metabolism pathway. The metabolites elevated in BAV patients with higher baseline Vmax were labeled by letter $\mathrm{V}$. Those elevated in BAV patients with $\triangle \mathrm{LVMI}$ value within the $1^{\text {st }}$ quartile were labeled by letter $\mathrm{P}$ 


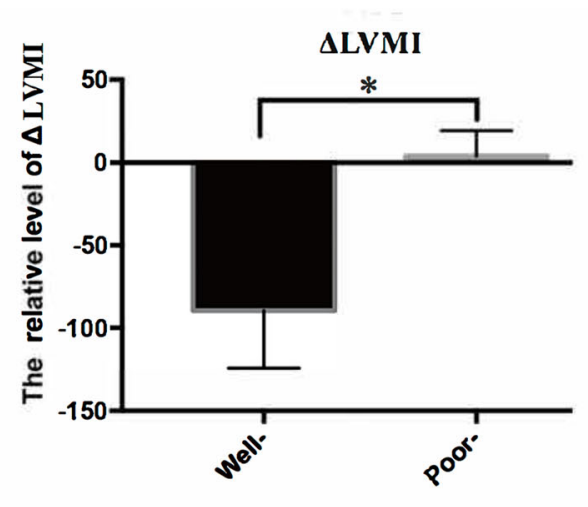

(A)

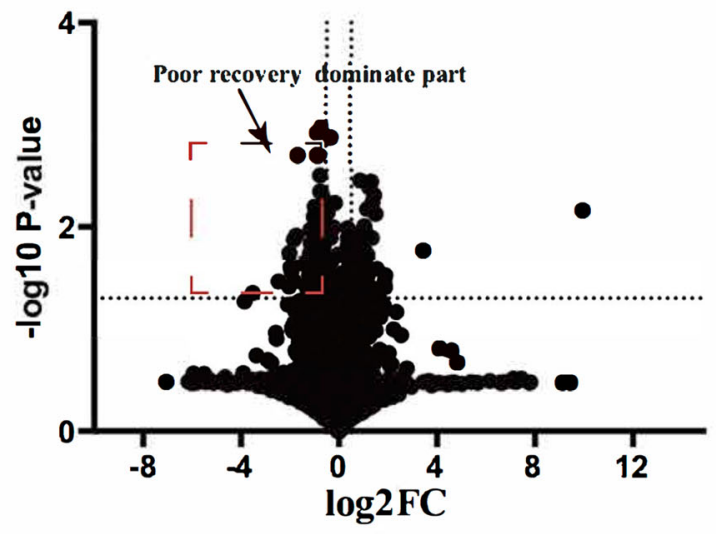

(C)

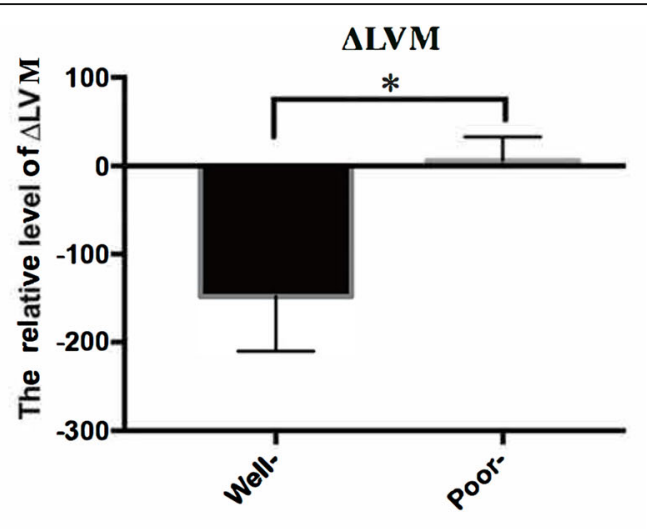

(B)

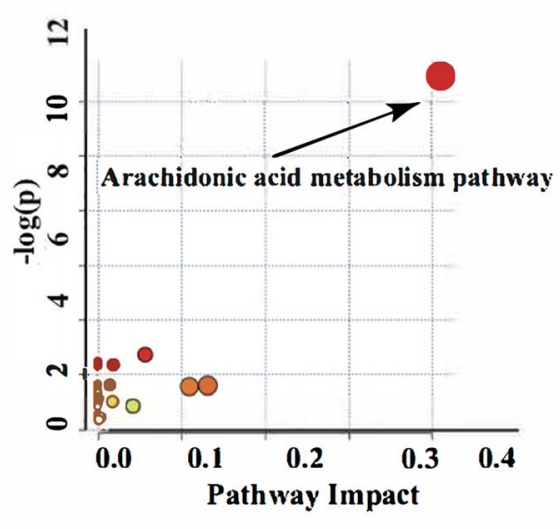

(D)
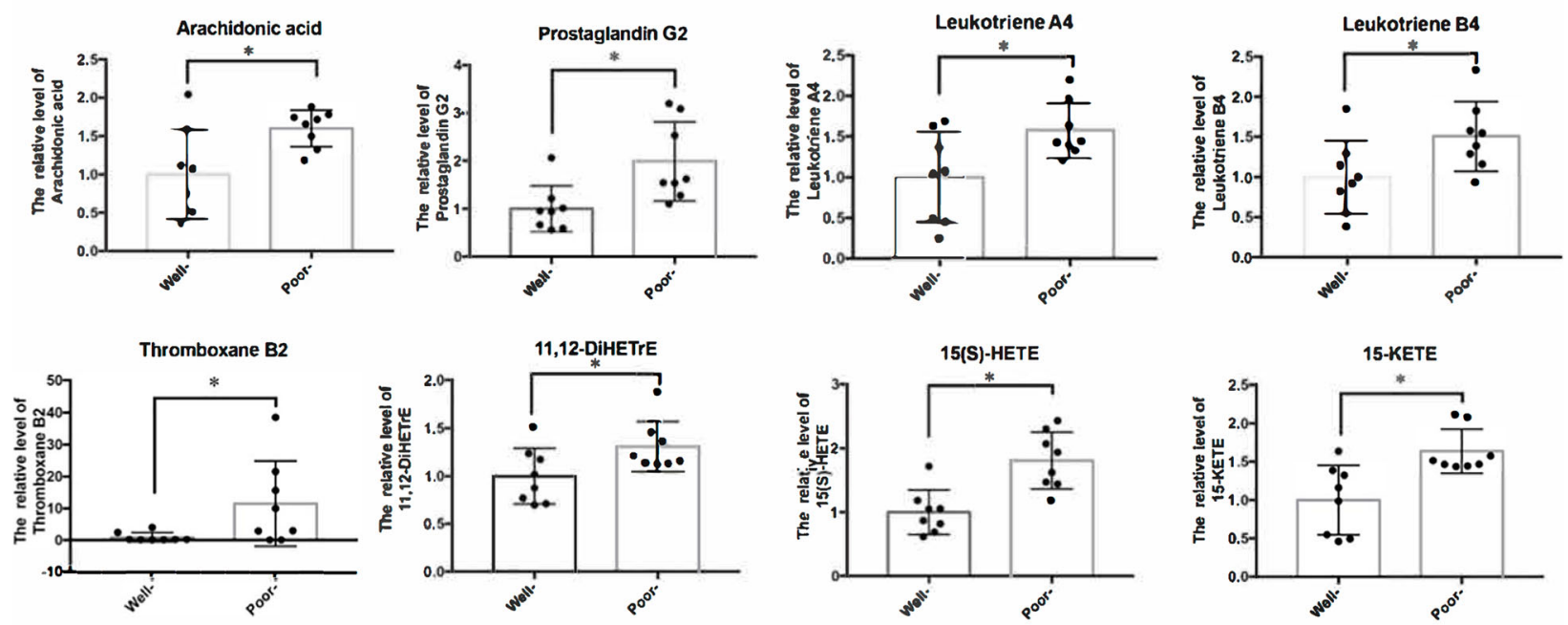

(E)

Fig. 6 Post-procedural subgroup analysis for BAV patients. a One-year $\triangle L V M I$ and (b) $\triangle L V M$ in the $1^{\text {st }}$ and $4^{\text {th }}$ quartile groups (all $P$-value $<0.05$ ). $\mathbf{c}$ Volcano plot showed the differentially expressed metabolites in the two groups. $\mathbf{d}$ The pathway enrichment analysis demonstrated elevated arachidonic acid metabolism pathway in patients within the $1^{\text {st }}$ quartile of $\Delta \mathrm{LVMI}(P$-value $<0.05)$. e The different expression of metabolites in arachidonic acid metabolism pathway between patients within the $1^{\text {st }}$ and $4^{\text {th }}$ quartile of one-year $\Delta \mathrm{LVMI}\left({ }^{*}\right.$ indicated $P$-value $\left.<0.05\right)$ 
oxidative stress, and promote oxidative damage [22, 23]. Besides key enzymes in arginine and proline metabolism pathway, the detected crucial amino acids such as glutamine and proline, likewise, have biological significance for bicuspid aortic stenosis. Proline was previously reported to be the balancer of over oxidation, which was engaged in stabilizing proteins and antioxidant enzymes, and directly scavenging ROS [24]. A study has also demonstrated that glutamine effectively controlled systemical inflammatory reactions, especially the molecules of IL-1, IL-6, IL-8, and IL-10 [25], which might be the proposed factors for accelerating valve stenosis [26]. Thus, targeting eNOS or the key amino acids in arginine and proline metabolism pathway might be potential targets to delay the progression of bicuspid aortic stenosis. Previously, perturbations in fatty acid metabolism has been identified in calcific coronary artery disease stratified by the severity of calcification $[27,28]$. As a pathology with some similar characteristics, calcific aortic valve disease might share these alterations. However, in this analysis, BAV patients did not achieve a statistically higher calcium burden on the leaflets than TAV patients and the coronary calcium score was not assessed, making it difficult to evaluate the impact of total calcium burden on metabolomic profiling.

In the BAV subgroup analysis, both more severely affected baseline hemodynamics and poorly recovered LV postTAVR were linked to elevated arachidonic acid metabolism pathway. As an important pathway for inflammation, the alleviative arachidonic acid metabolism might be crucial to halt the pathologic progression of aortic stenosis, especially for BAV patients $[29,30]$. Within this pathway, the key enzyme of COX-2 was considered as an important drive for aortic stenosis by increasing valvular calcification and enhancing osteogenic genes including OPN and Runx2 [29]. In addition, COX-2 was reported to be central in the AngIIinduced macrophage recruitment and the expression of TNF- $\alpha$ of endothelial cells, which blockades macrophage infiltration and exaggerates expression of pro-inflammatory cytokines [30]. Although there was no research reporting the role of arachidonic acid metabolism pathway in the recovery phase after aortic valve replacement, it was otherwise demonstrated to influence myocardial functions. Targeting metabolites such as cyclooxygenases and leukotrienes B4 in the arachidonic acid metabolism pathway protected against heart failure, decreased myocardial fibrosis and rescued myocardial function [31, 32]. The involved mechanisms might lie in preserving microcirculation and limiting inflammation, such as inhibiting TNF- $\alpha$-mediated pathway and inflammatory mediators [33, 34]. Thus, targeting arachidonic acid metabolism pathway might be of tremendous therapeutic value in clinical practice and merits further detailed researches.
There were several limitations in this study. Firstly, blood samples were sent for metabolomics detection without particular filtering. Secondly, there was gender imbalance possibly by coincidence at the time of this study, but cautions should be taken when interpreting the difference of metabolomic profiling between the two valve morphologies due to this selection bias. Thirdly, it was difficult to draw definitive conclusions due to relatively small sample size without a validation cohort and limited related researches in this area. A larger metabolomics study combining validation experiments should be arranged to verify our findings and explore the detailed mechanisms of molecules involved in bicuspid aortic stenosis.

\section{Conclusions}

This study comprehensively conducted metabolomics profiling for bicuspid aortic stenosis in the setting of TAVR, in comparison with TAV. Essential molecules in the arginine and proline metabolism pathway might delay the fast progression of bicuspid aortic stenosis. Metabolites in the arachidonic acid metabolism pathway might be potential biomarkers to dertermine BAV patients who would suffer from worse baseline hemodynamic stress or compromised LV recovery postTAVR. Further investigations are needed to validate these findings and explore underlying biological functions for these influential metabolic pathways.

\section{Supplementary information}

Supplementary information accompanies this paper at https://doi.org/10. 1186/s12872-020-01491-4.

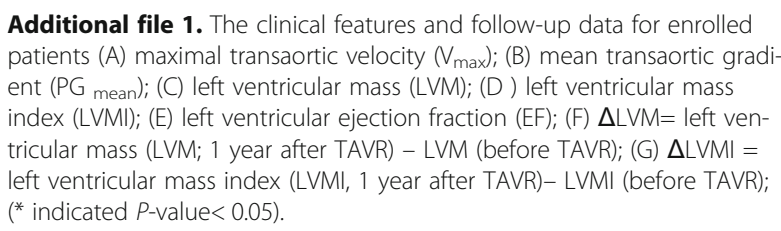

\section{Abbreviations}

BAV: Bicuspid aortic valve; TAV: Tricuspid aortic valve; LV: Left ventricle; TAVR: Transcatheter aortic valve replacement; MSCT: Multi-slice computed tomography; TTE: Transthoracic echocardiography; LVM: LV mass (LVM); LVMI: LV mass index; LC-MS: Liquid chromatography-mass spectrometry; GCMS: Gas chromatography-mass spectrometry; QC: Quality control;

SD: Standard deviation; PLS-DA: Partial least squares discriminant analysis; VIP: Variable importance project; FC: Fold change; WGCNA: Weighted correlation network analysis; NOS: Nitric oxide synthase

\section{Acknowledgements}

We thank Prof. Rong Tian from Mitochondria and Metabolism Center, University of Washington for her kind suggestions in data analysis and drafting of the manuscript.

Authors' contributions

$M C$ and $X J L$ conceived and designed research. YBL conducted experiments. YJL, YO, ZJW, XW, XL and YF recruited patients, collected raw data and participated in data interpretation. CL and WZ did statistical analysis. TYX and 
$\mathrm{CL}$ wrote the manuscript. CML, ZGZ and YF made critical revision sugesstions to the manuscript. TYX and YBL revised the manuscript. All authors read and approved the final manuscript.

\section{Funding}

This work was supported by the National Natural Science Foundation of China (81970325) for the design of the study; Science and Technology Support Plan of Sichuan province (2018SZ0172) for data analysis and interpretation; West China Hospital "1.3.5" Discipline of Excellence Project-Percutaneous transcatheter aortic valve implantation for the collection of data.

\section{Availability of data and materials}

The data are available from the corresponding author on reasonable request.

\section{Ethics approval and consent to participate}

This study was approved by the Institutional Review Board of West China Hospital, Sichuan University. Written informed consent was obtained from all patients being included in the study.

\section{Consent for publication}

Not applicable.

\section{Competing interests}

The authors have no conflicts of interests relevant to this article to be disclosed.

\section{Author details}

'Department of Cardiology, West China Hospital, Sichuan University, \#37 Guo Xue Alley, Chengdu 610041, People's Republic of China. ' Laboratory of Mitochondrial Biology, West China-Washington Mitochondria and Metabolism Center, West China Hospital, Sichuan University, Chengdu, People's Republic of China. '3aboratory of Cardiovascular Diseases, Regenerative Medicine Research Center, West China Hospital, Sichuan University, 610041 Chengdu, People's Republic of China.

Received: 29 January 2020 Accepted: 19 April 2020

Published online: 18 May 2020

\section{References}

1. Liu T, Xie M, Lv Q, Li Y, Fang L, Zhang L, et al. Bicuspid aortic valve: an update in morphology, genetics, biomarker, complications, imaging diagnosis and treatment. Front Physiol. 2018;9:1921.

2. Pedersen MW, Groth KA, Mortensen KH, Brodersen J, Gravholt CH, Andersen $\mathrm{NH}$. Clinical and pathophysiological aspects of bicuspid aortic valve disease. Cardiol Young. 2019;29:1-10

3. Kanjanahattakij N, Horn B, Vutthikraivit W, Biso SM, Ziccardi MR, Lu MLR, et al. Comparing outcomes after transcatheter aortic valve replacement in patients with stenotic bicuspid and tricuspid aortic valve: a systematic review and meta-analysis. Clin Cardiol. 2018;41:896-902.

4. Xiong T-Y, Wang X, Li Y-J, Liao Y-B, Zhao Z-G, Wei X, et al. Less pronounced reverse left ventricular remodeling in patients with bicuspid aortic stenosis treated with transcatheter aortic valve replacement compared to tricuspid aortic stenosis. Int J Cardiovasc Imaging. 2018;34:1761-7.

5. Jiao J, Tian W, Qiu P, Norton EL, Wang MM, Chen YE, et al. Induced pluripotent stem cells with NOTCH1 gene mutation show impaired differentiation into smooth muscle and endothelial cells: Implications for bicuspid aortic valverelated aortopathy. J Thorac Cardiovasc Surg. 2018;156:515-522.e1.

6. Giusti B, Sticchi E, De Cario R, Magi A, Nistri S, Pepe G. Genetic bases of bicuspid aortic valve: the contribution of traditional and high-throughput sequencing approaches on research and diagnosis. Front Physiol. 2017;8:612.

7. Mathieu P, Boulanger M-C. Autotaxin and lipoprotein metabolism in calcific aortic valve disease. Front Cardiovasc Med. 2019;6:18.

8. Tang WHW, Kitai T, Hazen SL. Gut microbiota in cardiovascular health and disease. Circ Res. 2017;120:1183-96.

9. Villas-Bôas SG, Rasmussen S, Lane GA. Metabolomics or metabolite profiles? Trends Biotechnol. 2005;23:385-6

10. Liao Y, Li Y, Xiong T, Ou Y, Lv W, He J, et al. Comparison of procedural, clinical and valve performance results of transcatheter aortic valve replacement in patients with bicuspid versus tricuspid aortic stenosis. Int J Cardiol. 2018;254:69-74.

11. Lang RM, Badano LP, Mor-Avi V, Afilalo J, Armstrong A, Ernande L, et al. Recommendations for cardiac chamber quantification by echocardiography in adults: an update from the American Society of Echocardiography and the European Association of Cardiovascular Imaging. J Am Soc Echocardiogr Off Publ Am Soc Echocardiogr. 2015;28:1-39.e14.

12. Liu Z, Yin P, Amathieu R, Savarin P, Xu G. Application of LC-MS-based metabolomics method in differentiating septic survivors from non-survivors. Anal Bioanal Chem. 2016:408:7641-9.

13. Zhou L, Wang Z, Hu C, Zhang C, Kovatcheva-Datchary P, Yu D, et al. Integrated metabolomics and Lipidomics analyses reveal metabolic reprogramming in human Glioma with IDH1 mutation. J Proteome Res. 2019;18:960-9.

14. Reimand J, Isserlin R, Voisin V, Kucera M, Tannus-Lopes C, Rostamianfar A, et al. Pathway enrichment analysis and visualization of omics data using $\mathrm{g}$ : profiler, GSEA, cytoscape and enrichment map. Nat Protoc. 2019;14:482-517.

15. Ding M, Li F, Wang B, Chi G, Liu H. A comprehensive analysis of WGCNA and serum metabolomics manifests the lung cancer-associated disordered glucose metabolism. J Cell Biochem. 2019;120:10855-63.

16. Chong J, Soufan O, Li C, Caraus I, Li S, Bourque G, et al. MetaboAnalyst 4.0: towards more transparent and integrative metabolomics analysis. Nucleic Acids Res. 2018:46:W486-94.

17. Michelena HI, Desjardins VA, Avierinos J-F, Russo A, Nkomo VT, Sundt TM, et al. Natural history of asymptomatic patients with normally functioning or minimally dysfunctional bicuspid aortic valve in the community. Circulation. 2008;117:2776-84.

18. Ziolo MT, Kohr MJ, Wang H. Nitric oxide signaling and the regulation of myocardial function. J Mol Cell Cardiol. 2008:45:625-32.

19. Massion PB, Balligand J-L. Relevance of nitric oxide for myocardial remodeling. Curr Heart Fail Rep. 2007;4:18-25.

20. Aicher D, Urbich C, Zeiher A, Dimmeler S, Schäfers H-J. Endothelial nitric oxide synthase in bicuspid aortic valve disease. Ann Thorac Surg. 2007:83:1290-4.

21. Kotlarczyk MP, Billaud M, Green BR, Hill JC, Shiva S, Kelley EE, et al. Regional disruptions in endothelial nitric oxide pathway associated with bicuspid aortic valve. Ann Thorac Surg. 2016;102:1274-81.

22. Shimokawa H, Godo S. Diverse functions of endothelial NO synthases system: NO and EDH. J Cardiovasc Pharmacol. 2016;67:361-6.

23. Godo S, Shimokawa H. Divergent roles of endothelial nitric oxide synthases system in maintaining cardiovascular homeostasis. Free Radic Biol Med. 2017;109:4-10.

24. Liang X, Zhang L, Natarajan SK, Becker DF. Proline mechanisms of stress survival. Antioxid Redox Signal. 2013;19:998-1011.

25. Lin Y-R, Li C-J, Syu S-H, Wen C-H, Buddhakosai W, Wu H-P, et al. Early Administration of Glutamine Protects Cardiomyocytes from post-cardiac arrest acidosis. Biomed Res Int. 2016;2016:1-8.

26. Manno G, Bentivegna R, Morreale P, Nobile D, Santangelo A, Novo S, et al. Chronic inflammation: a key role in degeneration of bicuspid aortic valve. J Mol Cell Cardiol. 2019;130:59-64.

27. Vorkas PA, Isaac G, Holmgren A, Want EJ, Shockcor JP, Holmes E, et al. Perturbations in fatty acid metabolism and apoptosis are manifested in calcific coronary artery disease: an exploratory lipidomic study. Int J Cardiol. 2015;197:192-9.

28. Djekic D, Pinto R, Vorkas PA, Henein MY. Replication of LC-MS untargeted lipidomics results in patients with calcific coronary disease: an interlaboratory reproducibility study. Int J Cardiol. 2016;222:1042-8.

29. Wirrig EE, Gomez MV, Hinton RB, Yutzey KE. COX2 inhibition reduces aortic valve calcification in vivo. Arterioscler Thromb Vasc Biol. 2015;35:938-47.

30. Avendaño MS, Martínez-Revelles S, Aguado A, Simões MR, González-Amor $M$, Palacios R, et al. Role of COX-2-derived PGE 2 on vascular stiffness and function in hypertension: COX-2 and vascular remodelling in hypertension. Br J Pharmacol. 2016;173:1541-55.

31. Wang Y, Li C, Liu Z, Shi T, Wang Q, Li D, et al. DanQi pill protects against heart failure through the arachidonic acid metabolism pathway by attenuating different cyclooxygenases and leukotrienes B4. BMC Complement Altern Med. 2014;14:67.

32. Wang J, Lu L, Wang Y, Wu Y, Han J, Wang W, et al. Qishenyiqi dropping pill attenuates myocardial fibrosis in rats by inhibiting RAAS-mediated arachidonic acid inflammation. J Ethnopharmacol. 2015;176:375-84.

33. Saito Y, Watanabe K, Fujioka D, Nakamura T, Obata J, Kawabata K, et al. Disruption of group IVA cytosolic phospholipase $a_{2}$ attenuates myocardial ischemia-reperfusion injury partly through inhibition of TNF-a-mediated pathway. Am J Physiol-Heart Circ Physiol. 2012;302:H2018-30.

34. Zhu L, Xu C, Huo X, Hao H, Wan Q, Chen H, et al. The cyclooxygenase-1/ mPGES-1/endothelial prostaglandin EP4 receptor pathway constrains myocardial ischemia-reperfusion injury. Nat Commun. 2019;10:1888.

\section{Publisher's Note}

Springer Nature remains neutral with regard to jurisdictional claims in published maps and institutional affiliations. 